\title{
Performance Efficiency of Quantization using HSV Colour Space and Intersection Distance in CBIR
}

\author{
S.Niranjanan \\ Asst. Prof., Computer Science Department, \\ Mohamed Sathak College of Arts and Science, \\ Sholinganallur, Chennai-600 119
}

\author{
S.P.Raja Gopalan \\ Prof., Dept. of Computer Science \& Engineering, \\ GKM College of Engineering \& Technology, \\ Chennai -600063
}

\begin{abstract}
Content Based Image Retrieval (CBIR) is an active research field in the past decades. Against the traditional system where the images are retrieved based on the key word search, CBIR systems retrieve the images based on the visual content. Even though some of the modern systems like relevance feedback system which improves the performance of CBIR systems exists, the importance of retrieving the images based on the low level features like Colour, Texture and Shape still determine the development of CBIR systems and cannot be undermined. Colour Histograms, Histogram Distance Measurements, Colour Spaces and Quantization play an important role in retrieving images based on similarities. In this paper, we present a novel method for determining the efficiency of different quantization methods using HSV Colour space and measuring the Intersection distance of the images with a uniform size of 256 X 256 pixels for efficient image retrieval and comparing the time utilized for retrieval
\end{abstract}

\section{General Terms}

Content Based Image Retrieval (CBIR).

\section{Keywords}

Content Based Image Retrieval (CBIR), HSV Colour space, Intersection distance, quantization.

\section{INTRODUCTION}

Content Based Image Retrieval (CBIR) describes the content of the image using the visual features like Colour, Texture and Shape. It retrieves the images based on the visual contents rather than the traditional model of key word annotations of the contents. Even though it has many advantages, It also has the major problem known as semantic Gap. In the semantic Gap, the description of the images using the low level features is unable to match the semantic intended by the user in his/her queries. Thus large number of false positive images are retrieved by the CBIR systems. The number of false positives are reduced when the spatial distribution of the visual features are integrated[1]. Even though some of the modern systems like relevance feedback system are introduced to improve the performance of CBIR exists, the importance of retrieving the images based on the low level features like Colour, Texture and Shape still determine the development of CBIR systems and cannot be undermined. In many cases they are the bottlenecks for development of CBIR techniques. A very basic issue in designing a CBIR system is to select the most effective image features for representing the image contents. Colour, Texture and Shape features are of the important and great majority primitive image descriptors in CBIR systems. Colour feature is one of the most reliable and easier visual features used in Image retrieval. It is robust to background complications and is independent of image size and orientation [16]. A lot of techniques available for retrieving images on the basis of Colour similarity from image database [3]. Colour Spaces, Colour Histograms, Histogram Distance Measurements, Size and Quantization play an important role in retrieving images based on similarities.

\section{COLOUR HISTOGRAM}

A colour histogram is a high dimensional feature vector, having greater than 100 dimensions. The comparison of histograms is computationally intensive. The colour histogram is widely used as an important colour feature indicating the contents of the images in content-based image retrieval (CBIR) systems. Especially, histogram-based algorithms are considered to be more effective for colour image indexing.

The global distribution of pixels of an image is described in Colour histogram, and it is insensitive to variations in scale and easy to calculate. The colour histogram for an image is constructed by counting the number of pixels of each colour. There are many difficulties with histogram based retrieval. The first of these is the high dimensionality of the colour histograms. Even with drastic quantization of the colour space, the image histogram feature spaces can occupy over 100 dimensions in real valued space. The large dimensionality also increases the complexity and computation of the distance function

An image histogram refers to the probability mass function of the image intensities. This is extended for Colour images to capture the joint probabilities of the intensities of the three Colour channels. More formally, the Colour histogram is defined by

$\mathrm{h}_{\mathrm{A}, \mathrm{B}, \mathrm{C}}(\mathrm{a}, \mathrm{b}, \mathrm{c})=\mathrm{N} \cdot \operatorname{Prob}(\mathrm{A}=\mathrm{a}, \mathrm{B}=\mathrm{b}, \mathrm{C}=\mathrm{c})$

where $A, B$ and $C$ represent the three Colour channels (R,G,B or $\mathrm{H}, \mathrm{S}, \mathrm{V})$ and $N$ is the number of pixels in the image. Computationally, the Colour histogram is formed by discretizing the Colours within an image and counting the number of pixels of each Colour.

Substantial quantization of the Colour space is needed, since the typical computer represents Colour images with up to 224 Colours. The main issues regarding the use of Colour histograms for indexing involve the choice of Colour space and correct quantization of the Colour space. When a perceptually uniform Colour space is chosen uniform quantization may be appropriate. If a non-uniform Colour space is chosen, then non-uniform quantization may be needed. 
The HSV Colour space offers improved perceptual uniformity. It represents with equal emphasis the three Colour variants that characterize Colour: Hue, Saturation and Value (Intensity). This separation is attractive because Colour image processing performed independently on the Colour channels does not introduce false Colours.

There are several distance formulas for measuring the similarity of Colour histograms. The Colour distance formulas arrive at a measure of similarity between images based on the perception of Colour content. Three distance formulas that are used normally for image retrieval are histogram euclidean distance, histogram intersection distance and histogram quadratic (cross) distance [11].

\section{HISTOGRAM INTERSECTION DISTANCE}

Let $h$ and $g$ represent two Colour histograms. The intersection of histograms $\boldsymbol{h}$ and $\boldsymbol{g}$ is given by:

$$
d(h, g)=\frac{\sum_{A} \sum_{B} \sum_{C} \min (h(a, b, c), g(a, b, c))}{\min (|h|,|g|)}
$$

where $|\boldsymbol{h}|$ and $|\boldsymbol{g}|$ gives the magnitude of each histogram, which is equal to the number of samples. Colors not present in the user's query image do not contribute to the intersection distance. This reduces the contribution of background colors. The sum is normalized by the histogram with fewest samples.

Count the number of pixels that correspond to a specific Colour in quantized Colour space for computation of histogram. In order to compare histograms of two images, we first need to generate specific codes for all histogram bins.

\section{COLOUR SPACE QUANTIZATION}

A Color quantization is a process that reduces the number of distinct colors used in an image. The intention of Colour quantization is that the new image should be as visually similar as possible to the original image. For a true color image, the number of the kind of colors are up to $2^{24}=$ 16777216 , so the direct extraction of color feature from true color will lead to a large computation. In order to reduce the computation, without a significant reduction in image quality, some representative color is extracted, to represent the image, thereby reducing the storage space and enhancing the process speed [5]. The effect of color quantization on the performance of image retrieval has been reported by many authors in [12] [14] [15] and [18], with different quantization schemes, like RGB (8X8X8), Lab (4X8X8), HSV (16X4X4), Lu*v* (4X8X8).

Manimala Singlia [8] concluded that, the HSV quantization $(16,4,4)$ with Histogram Intersection Distance gives best performance then the other colour spaces and the average retrieval time of HSV Colour space gives the optimum value, considering the precision and recall values. Bo Di [4] quantized HSV Colour space, 19 hues step $20(0,20,40, \ldots$, $360), 4$ saturations, step $0.25(0.173,0.423,0.673,0.923)$ and 5 values, step $0.25(0,0.25,0.5,0.75,1.00)$. Muhammad Riaz et al [9] defined: 18 hues, step $20(350,10,30,50 \ldots 350), 5$ saturations, step $0.2(0,0.2,0.4,0.6,0.8,1.0)$ and 3 values, steps $0.25(0,0.5,0.75,1.0)$. Ch. Kavitha et al [6],[7] defined 8 hues, step (316-20, 21-40, 41-75, 76-155, 156-190, 191-270, 271-295 and 296-315), 3 saturations, step (0-0.2, 0.2-0.7 and $0.7-1.0)$ and 3 values, step (0-0.2, 0.2-0.7 and 0.7-1.0) and concluded that colour and texture features of image sub blocks combined with normalized Euclidean distance yields better performance results. M.Babu Rao et al [2] defined 8 hues, 3 saturations and 3 values. Wasim Khan et al [17] defined 18 hues, 3 saturations and 3 values. Sangoh Jeong [11] used 18 hues, 3 saturations, 3 values and concluded that the Histogram Intersection-based image retrieval in HSV Colour space is most desirable among six retrieval methods including histogram euclidean distance in RGB Colour space, histogram intersection in RGB Colour Space, histogram quadratic (cross) distance in RGB Colour Space, histogram euclidean distance in HSV Colour space, histogram intersection in HSV Colour Space, histogram quadratic (cross) distance in HSV Colour Space mentioned in considering both computation time and retrieval effectiveness. Rajshree Dubey et al [10] have found that the histogram Euclidean distance and histogram intersection distance in HSV Colour space are most useful among histogram distance measures in the average sense. Vishal Chitkara [13] found that Variable-Bin Allocation (VBA) performs better than Constant-Bin Allocation (CBA) and the VBA approach not only performs better, but also consumes less resources, which is an important feature.

\section{PROPOSED MODEL}

\subsection{Methodology}

In this paper we address the issue of image database retrieval based on Colour using HSV colour space. Histogram search characterizes an image by its Colour distribution. Before extracting the maximum Colour from each segment the input image is converted to HSV and adaptive segmentation is applied on the HSV Colour space. This will compute the feature vector. Different quantization of hue, saturation and value are used. In this experiment, codes were generated for 10 different hue steps, 3 different saturations steps and 3 different value steps (10 H x $3 \mathrm{~S}$ x $3 \mathrm{~V}$ ) $90 \mathrm{HSV}$ histogram models. Accordingly codes were generated for more Histogram bins and then, histogram Intersection distances were computed in each quantized Colour space. Minkowski Form Distance Matrics is used to calculate the distance.

The corresponding image is first resized to $256 \mathrm{X} 256$ pixels, then converted from RGB to HSV Colour space using known equations, and then it is partitioned into $\mathrm{m}$ number of areas based on different ranges of hue, saturation and value. In the next step each area is partitioned into $\mathrm{n}$ number of segments based on the number of pixels it contains. After getting those segments we calculated the maximum Colour occurrence in each segment using the hue histogram information and this is used as a feature vector. Finally, the feature vector for query image is compared with the feature vector of the database images. The proposed system has been implemented and verified 


\subsection{Maximum Colour Selection}

The images are converted from RGB to HSV using the conversion formula is as follows

$H=\cos ^{-1}\left\{\frac{1 / 2[(R-G)+(R-B)]}{\sqrt{(R-G)^{2}+(R-B)(G-B)}}\right\}$

$S=1-\frac{3}{R+G+B}[\min (R, G, B)]$

$V=\frac{1}{3}(R+G+B)$

After the execution of these equations $\mathrm{H}, \mathrm{S}$ and $\mathrm{V}$ will have values $0-255$. To convert hue into angle that ranges from $0^{\circ}$ to $360^{\circ}$, value and saturation from 0 to 1 following equations are used

$\mathrm{H}=((\mathrm{H} / 255) * 360) \bmod 360$

$\mathrm{V}=\mathrm{V} / 255$

$\mathrm{S}=\mathrm{S} / 255$

This operation will make the computation easier. The goal of system is to reduce the Colour gamut and the insignificant Colour information in the image and emphasize the prominent Colour regions.

After conversion from RGB to HSV the next design parameter is the quantization of the Colour space. The HSV Colour space is cylindrical. The long axis represents value: blackness to whiteness. Distance from the axis represents saturation: amount of Colour present. The angle around the axis is the hue: tint or tone. Since hue represents the most significant characteristic of the Colour, it requires the finest quantization. In the hue circle the primaries red, green and blue are separated by 120 degrees.

We have tested a large number of images, and decided to verify which step could yield best results and made different steps in Hue, Saturation and Value. We have defined 10 Hue models with various steps which varies from 5 to 180 hues, Step $5(355,360,5,10,15, \ldots 355)$, Step 10 ( 350, 360, 10, $20, \ldots 350)$, Step $15(345,360,15,30, .345)$, Step 20 ( 340, $360,20,40, \ldots 340)$, Step $24(336,360,24,48, \ldots 336)$, Step $30(330,360,30,60, \ldots 330)$, Step $60(300,360,60, \ldots$ $360)$, Step $90(270,360,90, \ldots 270)$, Step $120(240,360$, $120,240)$ and Step 180 (180, 360, 180$)$. We have defined 3 Saturation models with various steps which varies from 2 to 10 Saturations, Step 0.5 ( 0, 0.5, 1.0$)$, Step $0.2(0,0.2,0.4$, $0.6,0.8,1.0)$ and Step $0.1(0,0.1,0.2, \ldots 1.0)$. We have defined 3 Values models with various steps which varies from 2 to 10 Values, Step 0.5 ( 0, 0.5, 1.0$)$, Step $0.2(0,0.2,0.4$, $0.6,0.8,1.0)$ and Step $0.1(0,0.1,0.2, \ldots 1.0$.

As a result we got $2 * 2 * 2=8$ to $72 * 10 * 10=7,200$ number of different areas, each representing different Colour distribution of the image. After partitioning into $m$ number of areas HSV information spaced image is further divided into $n$ number of segments depending upon the number of pixels in each area. The areas which have more pixels are partitioned into more number of segments and the areas which have fewer pixels are divided into less number of segments. Through this procedure we are able to get the distribution characteristics for the Colour tone distributed in the corresponding image. After extracting this Colour distribution information from the image we calculated the maximum Colour occurrence in each segment by using hue histogram of the image and HSV value of that pixel is used to make a feature vector. Such operations are carried out for the entire image and then feature vector is computed.

Following equation is used to partition each area in n number of segments; $\mathrm{n}$ can have different values for different areas.

$n_{i=\left(\left(X_{i} / T\right)\right) * T S} \quad 0<i<=m$

Where $\mathrm{n}_{\mathrm{i}}$ represents the number of segments in area i, $x_{i}$ represents the number of pixels in area $i$ (where $i$ ranges from $l$ to $m), T$ represents total number of pixels of the image and $T S$ represents total number of required segments of the entire HSV image. After executing the equation hue histogram is computed for each segment. From that histogram we can easily detect the each Colour occurrence in that segment. We have selected the maximum Colour occurrence from each segment and used its HSV value to compute the feature vector. This feature vector is used for comparison with the images in the database and matching for each image is calculated using Minkowski Metric (LM norm) equation. Segments in each area of the query image are compared with the corresponding area of the database image and for this comparison we used Minkowski Metric as shown below:

$d_{M(i, j)=\left(\sum_{k=1}^{P}\left|\left(x_{i}^{k}-x_{j}^{k}\right)\right|\right)^{1 / M}}$

Where, $\mathrm{p}$ is the dimension of the vector $x_{i}^{k}$ is the $k^{\text {th }}$ element of $x_{i}$. Three special cases of the LM metric are of particular interest, namely, $\mathrm{L}=1,2, \infty$.

A Matlab program using these different quantization schemes is programmed to retrieve images from a fixed size data base using a query image. The time consumed for displaying the resultant images is stored in a database along with the quantization variants data. The screen shots of retrieved images are also stored with appropriate names.

\subsection{Algorithm For Proposed Scheme}

Step 1: Load database in the Mat lab workspace.

Step 2: Resize the image for [256. 256].

Step 3: Convert image from RGB to HSV.

Step 4: Generate the histogram of hue, saturation and value

Step 5: Generate no. of signatures $n$ for hue, $m$ for saturation and $x$ for value.

Step6: Store the signature of database images into the mat file

Step 7: Load the Query image.

Step 8: Apply the procedure 2-7 to find signature of Query image.

Step 9: Determine the normalized Intersection distance of signature of Query image with stored signature of database.

Step 10: Sort the normalized Intersection distance values to perform indexing.

Step 11: Display the result on GUI.

Step 12: Create a folder in the name of corresponding variants

Step 13: Create a file in the name of corresponding variants \& save

Step 14: Apply the procedure 2-13 for various Hue, Saturation and Value steps 


\subsection{Performance Evaluation}

The performance of retrieval system can be measured in terms of its recall and precision. Recall measure the ability of the system to retrieve all the models that are relevant, while precision measures the ability of the system to retrieve only models that are relevant. This histogram has a high recall and precision of retrieval, and is effectively used in content-based image retrieval systems. They are defined as:

Precision $=\frac{\text { Number of relevant images retrieved }}{\text { Total number of images retrieved }}=\frac{A}{A+B}$

Precision $=\frac{\text { Number of relevant images retrieved }}{\text { Total number of relevant images }}=\frac{A}{A+C}$

Where A represent the number of relevant images that are retrieved, $\mathrm{B}$, the number of irrelevant items and the $\mathrm{C}$, number of relevant items those were not retrieved. The number of relevant items retrieved is the number of the returned images that are similar to the query image in this case. The number of relevant items in collection is the number of images that are in the same particular category with the query image. The total number of items retrieved is the number of images that are returned by the search engine.

\section{EXPERIMENTAL RESULTS}

We have purposefully kept only 7 relevant images of the particular query image type in the database folder along with other irrelevant images. We have designed a 3 X 3 matrix for showing the resultant retrieved images in the result window. Out of the total 9 images in the resultant matrix only 7 relevant images can be retrieved by the program and the other two images are selected based on the nearest Intersection distance. Hence the combination of different Hue, Saturation and Value steps which achieved retrieving all the 7 relevant images from the database folder in the least time is the best possible combination for image retrieval in HSV colour space.

Table 1 contains all the combinations of Hue, Saturation and Value steps which resulted in retrieving all the 7 possible relevant images from the database folder and whose Recall value is equal to 1 . It is also arranged in the increasing order of Time Utilized for the Retrieval process.

Since Table 1 only contains the best possible combinations of Hue, Saturation and Value steps, a Chart is plotted to visualize the effects of these steps against Time. Chart 1 visualizes the above effect. Since other data which doesn't produce the Recall value of 1 , and hence does not contain the best possible combinations of Hue, Saturation and Value, the data is not tabulated and no chart is required to visualize the effects and hence no Chart is plotted. The resultant image window of the least time consumed HSV slice $(2,2,10)$ in Table 1 is displayed along with the Quantization variations and time consumed.

Many researchers in their works adopted different Colour Space Quantizations like Ch.Kavitha et. Al. [6],[7] and M.Babu Rao et. Al. [2] with $(8,3,3)$, Manimala Singlia [8] with $(16,4,4)$, Wasim Khan et. al. [18] and Sangoh Jeong [11] with $(18,3,3)$, Mohammad Riaz et. al. [9] with $(18,5,3)$ and Bo Di [4] with $(19,4,5)$. Hence, the present work with the result of HSV Slice $(\mathbf{2 , 2 , 1 0 )}$ in HSV Colour Space Quantization with Intersection Distance with least time consumption gives a better alternative than other Quantization schemes.

\section{CONCLUSION}

The above results show that it can be possible to retrieve all the images of uniform size $256 \times 256$ pixels from the databases by adopting only 2 slices of Hue (i.e. 0 - 180 and $180-360$ ), 2 slices of Saturation (i.e. $0-0.5$ and $0.6-1.0$ ) and 10 slices of Value (i.e. $0-0.1,0.1-0.2,0.2-0.3,0.3-$ $0.4,0.4-0.5,0.5-0.6,0.6-0.7,0.7-0.8,0.8-0.9$ and 0.9 - 1.0). Also the results indicate that whenever we slice the Value component in to 10 slices, it yields the best results.

Thus $2 \times 2 \times 10=40$ slices split is more than sufficient to retrieve the images in much lesser Time. Thus it is concluded that the HSV colour space Quantization $(2,2,10)$ with Histogram Intersection distance under a uniform size of $256 \mathrm{X}$ 256 pixels gives better performance than other variations of Hue, Saturation and Value quantization models in image retrieval. Many Researchers in their works have adopted the HSV Colour Space Quantization schemes with $(8,3,3)$, $(16,4,4),(18,3,3),(18,5,3),(19,4,5)$ etc.,. Thus the present work with $(2,2,10)$ with least time is a better alternative in HSV Colour Space Quantization with Intersection Distance. 
HSV Experimental Results of Most Desirable Quantization Variants

Table 1 Results where Recall $=1$, Precision $=0.777778$ and retrieved all relevant images $(7)$

\begin{tabular}{|c|c|c|c|c|c|c|c|}
\hline $\begin{array}{l}\text { HUE } \\
\text { Step }\end{array}$ & $\begin{array}{l}\text { SATUR } \\
\text { ATION } \\
\text { Step }\end{array}$ & $\begin{array}{l}\text { VALUE } \\
\text { Step }\end{array}$ & $\begin{array}{l}\text { Hue, } \\
\text { Saturation } \\
\text { and Value } \\
\text { Slices }\end{array}$ & $\begin{array}{l}\text { TIME in } \\
\text { Secs }\end{array}$ & $\begin{array}{l}\text { Relevant } \\
\text { images } \\
\text { Retrived } \\
\text { (A) }\end{array}$ & $\begin{array}{l}\text { Irrelavant } \\
\text { Images } \\
\text { Retrieved } \\
\text { (B ) }\end{array}$ & $\begin{array}{l}\text { Relevant } \\
\text { Images not } \\
\text { Retrieved } \\
\text { (C) }\end{array}$ \\
\hline 180 & 0.5 & 0.1 & $2,2,10$ & 69.4564 & 7 & 2 & 0 \\
\hline 180 & 0.2 & 0.1 & $2,5,10$ & 94.9177 & 7 & 2 & 0 \\
\hline 120 & 0.5 & 0.1 & $3,2,10$ & 108.9754 & 7 & 2 & 0 \\
\hline 90 & 0.5 & 0.1 & $4,2,10$ & 124.6061 & 7 & 2 & 0 \\
\hline 120 & 0.2 & 0.1 & $3,5,10$ & 147.3692 & 7 & 2 & 0 \\
\hline 60 & 0.5 & 0.1 & $6,2,10$ & 160.2266 & 7 & 2 & 0 \\
\hline 90 & 0.2 & 0.1 & $4,5,10$ & 163.9276 & 7 & 2 & 0 \\
\hline 60 & 0.2 & 0.1 & $6,5,10$ & 195.6958 & 7 & 2 & 0 \\
\hline 180 & 0.1 & 0.1 & $2,10,10$ & 205.5844 & 7 & 2 & 0 \\
\hline 120 & 0.1 & 0.1 & $3,10,10$ & 243.8917 & 7 & 2 & 0 \\
\hline 30 & 0.5 & 0.1 & $12,2,10$ & 262.3385 & 7 & 2 & 0 \\
\hline 90 & 0.1 & 0.1 & $4,10,10$ & 272 & 7 & 2 & 0 \\
\hline 30 & 0.2 & 0.1 & $12,5,10$ & 296.4077 & 7 & 2 & 0 \\
\hline 24 & 0.5 & 0.1 & $15,2,10$ & 312.0879 & 7 & 2 & 0 \\
\hline 60 & 0.1 & 0.1 & $6,10,10$ & 336.4362 & 7 & 2 & 0 \\
\hline 24 & 0.2 & 0.1 & $15,5,10$ & 344.8115 & 7 & 2 & 0 \\
\hline 20 & 0.5 & 0.1 & $18,2,10$ & 364.3345 & 7 & 2 & 0 \\
\hline 20 & 0.2 & 0.1 & $18,5,10$ & 391.2559 & 7 & 2 & 0 \\
\hline 15 & 0.5 & 0.1 & $24,2,10$ & 470.7421 & 7 & 2 & 0 \\
\hline 15 & 0.2 & 0.1 & $24,5,10$ & 492.1369 & 7 & 2 & 0 \\
\hline 30 & 0.1 & 0.1 & $12,10,10$ & 527.9013 & 7 & 2 & 0 \\
\hline
\end{tabular}




\begin{tabular}{|r|r|r|r|r|r|r|r|}
\hline 24 & 0.1 & 0.1 & $15,10,10$ & 624.1631 & 7 & 2 & 0 \\
\hline 10 & 0.5 & 0.1 & $36,2,10$ & 672.4624 & 7 & 2 & 0 \\
\hline 10 & 0.2 & 0.1 & $36,5,10$ & 691.0684 & 7 & 2 & 0 \\
\hline 20 & 0.1 & 0.1 & $18,10,10$ & 720.2375 & 7 & 2 & 0 \\
\hline 15 & 0.1 & 0.1 & $24,10,10$ & 914.6463 & 7 & 2 & 0 \\
\hline 5 & 0.2 & 0.1 & $72,5,10$ & 1281.554 & 7 & 2 & 0 \\
\hline 5 & 0.5 & 0.1 & $72,2,10$ & 1290.102 & 7 & 2 & 0 \\
\hline 10 & 0.1 & 0.1 & $36,10,10$ & 1297.051 & 7 & 2 & \\
\hline 5 & 0.1 & 0.1 & $72,10,10$ & 2445.133 & 7 & & 0 \\
\hline
\end{tabular}

Figure 1 - Resultant image window of the least time consumed HSV slice $(2,2,10)$

A

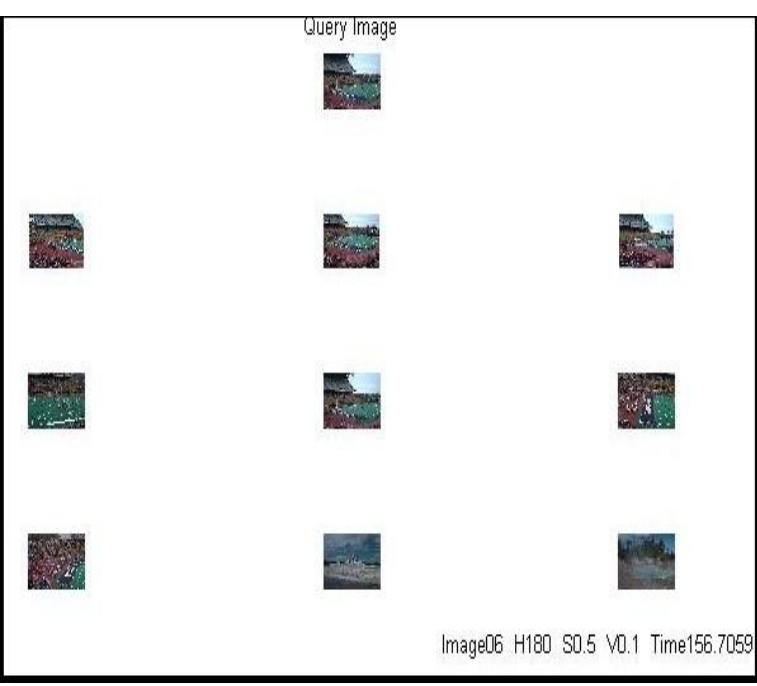

B
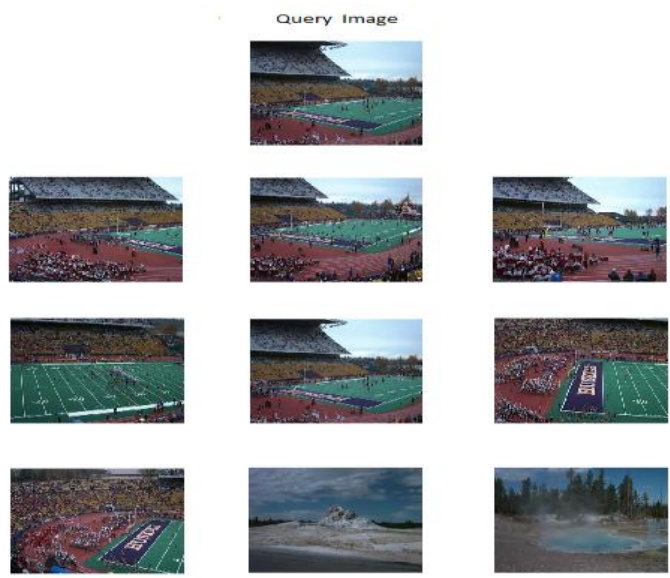

Image06 H180 So.5 Vo.1 Time156.7059

\section{A) Original Output Window from Program}

B) Images retrieved from the Database as shown in the Original Window displayed in order for Clarity 


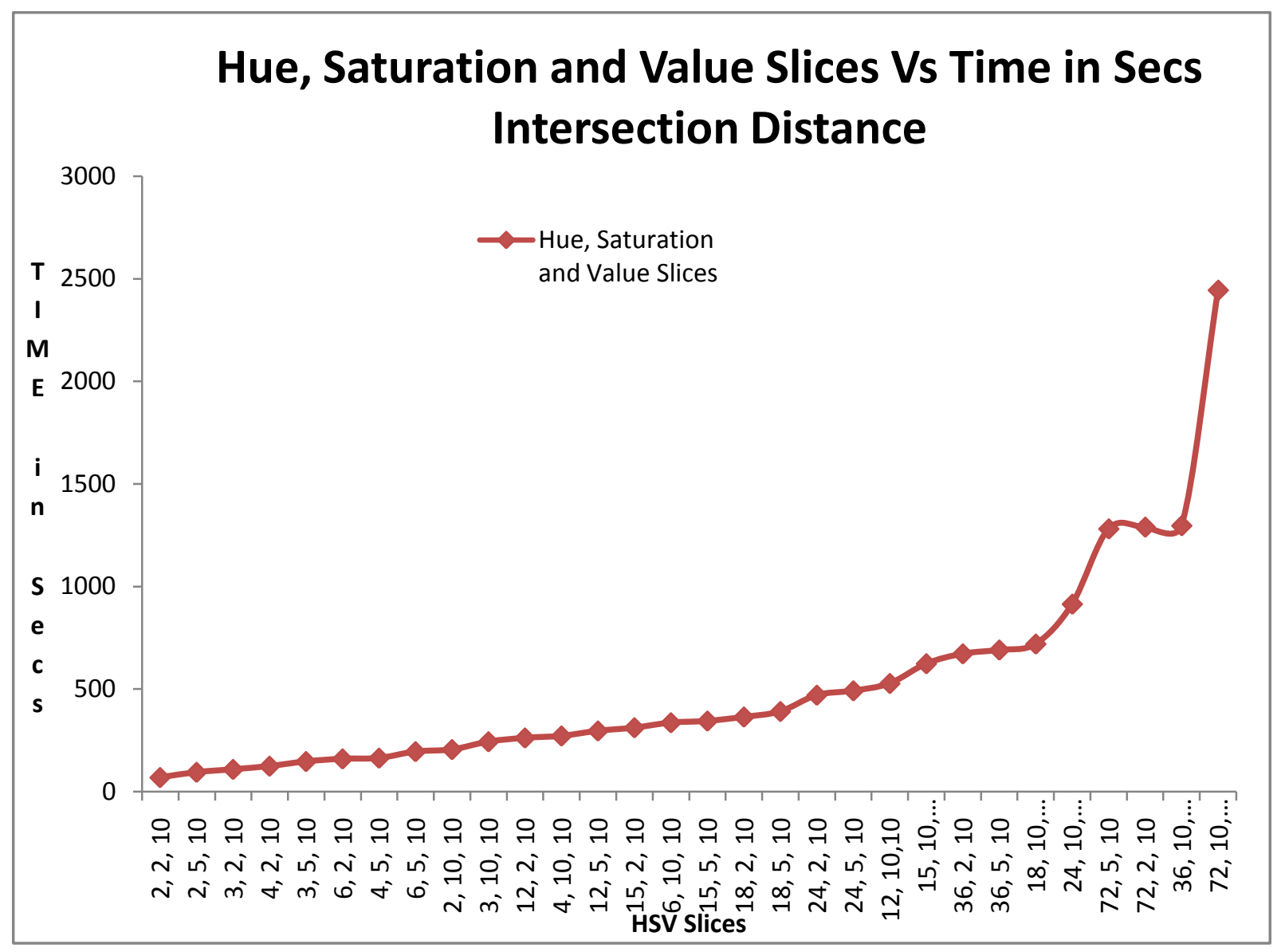

\section{REFERENCES}

[1] Abdel hamid Abdesselam, Hui Hui Wang, and Arayanan Kulathuramaiyer - "Spiral Bit-string Representation of Colour for Image Retrieval"

[2] M.Babu Rao, Dr. B.Prabhakara Rao \& Dr. A.Govardhan - Apr 2011 - "Content Based Image Retrieval Using Dominant Colour, Texture And Shape" - International Journal of Engineering Science and Technology (IJEST), Vol. 3 No. 4 ISSN : 0975-5462

[3] Bing Wang -2008 - "A Semantic Description For Content-Based Image Retrieval" - at College Of Mathematics And Computer Science, Hebei University, Baoding 071002, China

[4] Bo Di - 2007 - "An efficient image retrieval approach base on Colour clustering" at Third International Conference on Intelligent Information Hiding and Multimedia Signal Processing,. IIHMSP

[5] Hafner, J and Sawhney, H. S.- 1995 -. Efficient color histogram indexing for quadratic form distance functions. In IEEE Transactions on Pattern Analysis and Machine Intelligence, Intelligence, 17(7): pp.729736.

[6] Ch.Kavitha, Dr. B.Prabhakara Rao \& Dr. A.Govardhan Feb 2011 - "An Efficient Content Based image Retrieval Using Colour And Texture Of Image Subblocks" in International Journal of Engineering Science and Technology (IJEST), Vol. 3 No. 2, ISSN : 0975-5462
[7] Ch.Kavitha, Dr. B.Prabhakara Rao \& Dr. A.Govardhan February 2011.- "Image Retrieval Based On Colour and Texture Features of the Image Sub-blocks" in International Journal of Computer Applications (0975 8887)Volume 15- No.7,

[8] Manimala Singlia and K.Hemacllandran - 2011"Performance analysis of Colour Spaces In Image Retrieval" in Assam University Journal of Science \& Technology: Physical Sciences and Technology Vol. 7 Number 11 94-104. : ISSN 0975-2773

[9] Muhammad Riaz, Kim Pankoo and Park Jongan - 2009 "Extracting Colour Using Adaptive Segmentation for Image Retrieval" in International Joint Conference on Computational Sciences and Optimization

[10] Rajshree Dubey, Rajnish Choubey and Sanjeev Dubey June 2011 - "Efficient Image Mining using Multi Feature Content Based Image Retrieval System" in Int Jr of Advanced Computer Engineering and Architecture Vol. 1, No. 1 ,

[11] .Sangoh Jeong -Mar.15, 2001 - "Histogram-Based Colour Image Retrieval"

[12] .Smith, J. R. - 1997 -. Integrated spatial and feature image system: Retrieval, analysis and compression, Ph.D dissertation, Columbia University, New York

[13] Vishal Chitkara - May 2001 - "Colour-Based Image Retrieval Using Compact Binary Signatures" in Technical Report TR 01-08 Department Of Computing Science, University of Alberta Edmonton, Alberta, Canada 
[14] Wan, X and Kuo, - 1996 - Image retrieval with multiresolution color space quantization. in Electron.Imaging and Multimedia Syst.

[15] Wan. X and Kuo. K. - 1996 -. Color distribution analysis and quantization for image retrieval. in SPIE Storage and Retrieval for Image and Video Databases IV, vol.SPIE 2670, pp. 9- 16

[16] Waqas Rasheed - 2008 - "Sum of Values of Local Histograms for Image retrieval" at Chosun University, Gwangju, South Korea
[17] Wasim Khan, Shiv Kumar, Neetesh Gupta, Nilofar Khan - March 2011 - "Signature Based Approach For Image Retrieval Using Colour Histogram And Wavelet Transform" in International Journal of Soft Computing and Engineering (IJSCE) Volume-1, Issue-1

[18] Zhang, Z., Wenhui, Land Bo, L. - 2009 -. An Improving Technique of Col or Histogram in Segmentation-based Image Retrieval. At Fifih International Conference on Information Assurance and Security. IEEE. 